\title{
LA SOLIDARIDAD Y LAS DECLARACIONES INTERNACIONALES DE DERECHOS HUMANOS Y BIOÉTICA: EL CASO DEL DERECHO A LA SALUD DE LOS PUEBLOS INDÍGENAS COLOMBIANOS ${ }^{1}$
}

\author{
Diana Rocío Bernal Camargo ${ }^{2}$
}

Resumen: Este artículo muestra el papel del principio de solidaridad en relación con el derecho a la salud de los pueblos indígenas colombianos, a partir del análisis de la Declaración Universal de los Derechos de los Pueblos Indígenas y de la Declaración de Bioética y Derechos Humanos. Se presenta la situación actual de estos grupos étnicos en términos de acceso a los más altos niveles de salud, teniendo como base los informes de Naciones Unidas, los documentos oficiales de política pública colombiana, los lineamientos de Naciones Unidas y los postulados de los pueblos indígenas respecto del derecho a la salud. La revisión evidencia que el papel de la solidaridad en este campo debe servir como elemento de promoción de las prácticas tradicionales indígenas para integrarlas a los sistemas de salud occidentales. Esto garantizaría el acceso en equidad y el fortalecimiento de sus instituciones médicas, sin generar practicas de asimilación o exclusión en el acceso al derecho a la salud.

Palabras clave: solidaridad, bioética, derechos humanos, salud, pueblos indígenas

Solidarity, international human rights declarations and bioethics: the case of the right to health of aboriginal Colombian peoples

\begin{abstract}
This paper shows the role of the solidarity principle in relation to right to health of Colombian aboriginal peoples, analyzing the Universal declaration of the rights of aboriginal peoples and the Declaration on Bioethics and Human Rights. Current situation of these ethnic groups is presented as it relates to access to the highest levels of health, based on reports from United Nations and postulates of aboriginal peoples regarding the tight to health. The analysis evidences that the role of solidarity in this field should serve as an element for promoting traditional indigenous practices in order to integrate them to Western health systems. This would guarantee the access in equity and the strengthening of medical institutions without generating assimilation or exclusion in the access to the right to health.
\end{abstract}

Key words: solidarity, bioethics, human rights, health, aboriginal peoples

A solidariedade e as declaraçóes internacionais de direitos humanos e bioética: o caso do direito à saúde dos povos indígenas colombianos

Resumo: Este artigo mostra o papel do princípio da solidariedade em relação ao direito à saúde dos povos indígenas colombianos, a partir da análise da Declaraçáo Universal dos Direitos dos Povos Indígenas e da Declaração de Bioética e Direitos Humanos. Apresenta-se a situação atual destes grupos étnicos em termos de acesso aos mais altos níveis de saúde, tendo como base os informes das Naçóes Unidas, os documentos oficiais de política pública colombiana, as orientaçóes das Naçóes Unidas e os postulados dos povos indígenas em relação ao direito à saúde.

A revisão evidencia que o papel da solidariedade neste campo deve servir como elemento de promoção das práticas tradicionais indígenas para integrá-las aos sistemas de saúde ocidentais. Isto garantiria o acesso em equidade e o fortalecimento de suas instituiçốes médicas, sem gerar práticas de assimilação ou exclusão no acesso ao direito à saúde.

Palavras-chave: solidariedade, bioética, direitos humanos, saúde, povos indígenas

\footnotetext{
${ }^{1}$ Artículo que es parte del proyecto "Perspectiva bioética de la salud y su relación con otros derechos humanos en países en vía de desarrollo", adscrito a la línea de investigación "Políticas Públicas Sociales y Seguimiento Normativo", del Grupo de Investigación en Derechos Humanos, Facultad de Jurisprudencia, Universidad del Rosario.

${ }^{2}$ Grupo de Investigación en Derechos Humanos, Facultad de Jurisprudencia, Universidad del Rosario, Bogotá, Colombia

Correspondencia:diana.bernalc@urosario.edu.co
} 


\section{Introducción}

El reconocimiento de los pueblos indígenas (PIs) en diferentes regiones del mundo ha sido un proceso progresivo que ha permitido identificar sus necesidades en materia de protección de derechos humanos y señalar las deficiencias de las legislaciones nacionales en la materia. Como resultado, se ha dado mayor importancia a la preocupación sobre la garantía y disfrute de los derechos de los PIs y se les ha incluido dentro de la categoría de grupos vulnerables de especial protección, razón por la cual resulta especialmente pertinente el análisis de su situación respecto del goce del derecho a la salud. Se puede afirmar que el Estado desconoce el rol del principio bioético de la solidaridad, ya que las políticas de salud no se construyen tomando en cuenta la propia voz de los PIs, que resulta de suma utilidad para contribuir en el fortalecimiento de los sistemas de salud en el marco del respeto por la diversidad y el pluralismo.

Se requiere un escenario en el que los profesionales de la salud tengan un enfoque cultural para la atención de pacientes pertenecientes a pueblos étnicos, pero que a su vez se busque afianzar el sistema de medicina tradicional. En este sentido, sería pertinente indagar cómo el principio bioético de solidaridad está vinculado a la aplicación del derecho a la salud enunciado en el artículo 12 del Pacto Internacional de Derechos Económicos, Sociales y Culturales. La respuesta parte de los postulados que se encuentran en los instrumentos internacionales relacionados con esta temática, cuales son, la Declaración Universal de los Derechos de los Pueblos Indígenas (DUDPI) y la Declaración Universal de Bioética y Derechos Humanos (DUBDH), en consonancia con el Pacto Internacional de Derechos Económicos, Sociales y Culturales (PIDESC).

El objetivo es analizar el principio de solidaridad en los instrumentos en mención, en relación con el derecho al disfrute de los más altos niveles de salud de los PIs, teniendo en cuenta los informes y documentos que dan cuenta de su realidad.

Como método de trabajo, se realizó una revisión y análisis de declaraciones, informes y reportes de relatores especiales de Naciones Unidas, enfocados en la situación del acceso a la salud de los pueblos indígenas, para contrastarlos con el contenido y alcance del principio de la solidaridad como herramienta para la promoción de su derecho al acceso a la salud. También se revisaron artículos y documentos sobre el principio de solidaridad referidos a este derecho, en los que han participado representantes de los PIs colombianos.

\section{El principio de la solidaridad en DUBDH}

Para el análisis de este principio, se parte de la Declaración Universal de Bioética y Derechos Humanos, instrumento jurídico que proporciona un marco general de principios para que los Estados diseñen o complementen sus legislaciones y las políticas públicas en los diferentes campos de la bioética. Esta Declaración es un modelo hacia el consenso de normas globales en bioética, aunque en el proceso de su elaboración no se dieron las consultas necesarias y significativas con los Estados, tendientes a la consecución de una mayor recepción de esta norma en las legislaciones internas(1). Para tal fin, es prioritario que la comunidad internacional asuma las declaraciones en el campo de la bioética, no solo como normas éticas, sino como "soft law", específicamente como una extensión del Derecho Internacional de los Derechos Humanos en estas áreas, para que así adquieran carácter vinculante, a través de nuevos tratados, o por el camino de la creación normas consuetudinarias(2).

En este contexto, más que una norma bioética, la solidaridad es un principio que busca fortalecer el sistema de los Derechos Humanos, en especial si se tiene en cuenta que otros instrumentos internacionales de bioética ${ }^{3}$ lo han adoptado en su cuerpo normativo como principio que representa consenso acerca de este valor fundamental y es punto de referencia para las legislaciones internas.

En especial, interesan los artículos 13, 14 y 24 de la DUBDH(3), a partir de los cuales la solidaridad, junto con la cooperación, constituyen la

${ }^{3}$ Cfr. la Declaración Universal sobre el Genoma Humano y los Derechos Humanos, la Declaración Internacional sobre los Datos Genéticos Humanos o el Convenio Europeo sobre los Derechos Humanos y la Biomedicina. 
clave para el logro de los niveles más altos de salud, y deben aplicarse de manera interdependiente e integral, tanto con el texto de la Declaración como con el sistema internacional de Derechos Humanos(4).

De la lectura integral de la Declaración no se desprende una definición de la solidaridad, pero se pueden deducir características de la misma. Así, del artículo 24 se infiere que este principio implica una especial consideración por los grupos vulnerables, que se fundamenta en las relaciones internas del grupo o en las relaciones externas de identificación con otros grupos(5). Acorde con la revisión realizada por Gunson, "la solidaridad consiste en la voluntad de tomar seriamente la perspectiva de otros y actuar para fortalecerla" (5). Esta definición resulta de especial interés, puesto que permite afirmar que, en el caso del derecho a la salud, implica la construcción y ejecución conjunta de los diferentes elementos de los sistemas de salud para alcanzar los más altos niveles de acceso a este derecho.

El sistema jurídico colombiano, por su parte, hace referencia a la solidaridad como principio dentro del sistema de seguridad social en salud, y la entiende como aquella "práctica del mutuo apoyo para garantizar el acceso y sostenibilidad a los servicios de Seguridad Social en Salud, entre las personas"(6). Sin embargo, este principio no es producto de la adopción de las normas internacionales sobre bioética por parte del derecho interno.

\section{El derecho a la salud de los pueblos indígenas en PIDESC y DUDPI}

El acceso a la salud es un derecho universal y no puede ser objeto de discriminación alguna. Su reconocimiento internacional se fundamenta en la Declaración Universal de Derechos Humanos (DUDH), que en el artículo 25 dispone que toda persona tenga derecho a un estándar de vida adecuado para la salud de sí mismo y de su familia. Esta disposición se complementa con el artículo 12 del Pacto Internacional de Derechos Económicos, Sociales y Culturales (PIDESC), que incluye el deber de los Estados en procura de asegurar la efectividad del derecho de toda persona al disfrute del más alto nivel posible de salud física y mental.

Si se toma en cuenta la Declaración de Viena, surgida con ocasión de los 50 años de la DUDH, se debe señalar que los llamados derechos económicos, sociales y culturales - como el derecho a la salud - deben entenderse de forma universal, integral, interdependiente e indivisible con el sistema general de derechos humanos. En consecuencia, los DESC son verdaderos derechos fundamentales, justiciables y exigibles, y no simples declaraciones de voluntad(7).

Del mismo modo, instrumentos como la Constitución de la Organización Mundial de la Salud (preámbulo), la Carta Social Europea (parte I, artículo 11), y el Protocolo Adicional a la Convención Americana sobre Derechos Humanos en materia de derechos económicos, sociales y culturales, conocido como "Protocolo de San Salvador" (artículo 10), incluyen el derecho a la salud como parte del sistema de derechos humanos.

En relación con los grupos vulnerables o excluidos, como es el caso de los PIs, para la plena realización del derecho a la salud se requiere de medidas especiales y acciones diferenciadas, como resultado del reconocimiento a la diversidad cultural y en el marco del pluralismo. Muestra de ello fue la adopción de la Declaración Universal de Derechos de los Pueblos Indígenas ${ }^{4}$, en la que se hace reconocimiento a los pueblos indígenas como pueblos titulares de derechos y se enuncian algunos de ellos. En relación con el derecho a la salud, se encuentran:

- Protección especial para su población.

- Derecho a participar en la determinación y elaboración de prioridades y estrategias en sus programas de salud y, de ser posible, administrarlos mediante sus propias instituciones.

- Derecho a sus propias medicinas tradicionales y a mantener sus prácticas de salud.

- Derecho de acceso, sin discriminación alguna, a todos los servicios sociales y de salud.

- Derecho a disfrutar del nivel más alto posible

\footnotetext{
${ }^{4}$ Aprobada por la Asamblea General de la ONU en su sesión No. 62 a través de la resolución A/RES/61/295 del 13 de septiembre de 2007
} 
de salud física y mental.

- Deber de los Estados de adoptar medidas para lograr la plena realización del derecho a la salud de los pueblos indígenas(8).

Este derecho se entiende como sustento de una vida digna e implica el derecho a disfrutar de las facilidades, bienes, servicios y condiciones necesarios para alcanzar el nivel más alto posible de salud física y mental, es decir, que su realización se procura en conjunto con los demás derechos inherentes al ser humano(9). Esto significa, como lo recalcan Celkis y Venckiene, que los individuos deben gozar de la protección contra amenazas externas para su goce, y se debe garantizar la disponibilidad de servicios de salud de calidad suficientes para la población, en especial tomando en cuenta las necesidades de los grupos más vulnerables(10).

Acorde con la DUDPI, los estándares más altos de calidad del derecho a la salud, además de cumplir con las directrices de la Organización Mundial de la Salud, la Organización Panamericana de la Salud y de la Observación No 14 del ECOSOC — disponibilidad, accesibilidad física y económica, aceptabilidad y calidad-(11), se deben construir y efectivizar a través de la incorporación de la perspectiva indígena en la formulación de políticas públicas de salud, lo cual se traduce en la necesidad de tomar en consideración las prácticas tradicionales de salud y de medicina tradicional de los PIs.

La situación del derecho a la salud de los PIs colombianos acorde con los informes de $\mathrm{Na}$ ciones Unidas

El reconocimiento internacional, regional e interno de las necesidades en materia de salud de los PIs ha sido objeto de diversos informes, recomendaciones y observaciones por parte de Naciones Unidas, entre los cuales vale resaltar los siguientes:

a. Informes del relator especial sobre el derecho de toda persona al disfrute del más alto nivel posible de salud física y mental

En general, en los informes se denota una preocu- pación por los asuntos relativos al consentimiento informado de los PIs, quienes "son especialmente vulnerables en las investigaciones médicas efectuadas en sus comunidades, a causa de los incentivos indebidos que se les ofrecen y de los graves desequilibrios de poder de que adolecen"(12). Asimismo, resaltan la necesidad de tomar en cuenta prácticas tradicionales, como el consentimiento colectivo, y la preferencia por determinados proveedores de servicios de salud que se ajustan a sus necesidades y tradiciones(12).

En el reporte de octubre de 2004, el relator expresó que existen marcadas disparidades entre las condiciones de salud de los indígenas y el resto de la población en diferentes países del mundo. Señaló que los pueblos indígenas tienen una expectativa menor de vida y están propensos cada vez más a enfermedades de transmisión sexual, debido a prácticas de explotación sexual hacia mujeres y niños de sus comunidades. Agregó el informe que, acorde con la información allegada, se evidenciaron prácticas discriminatorias por parte de profesionales de la salud, quienes carecen de formación y sensibilidad hacia las necesidades de los PIs. De igual forma, se da cuenta de la falta de servicios de calidad en idiomas indígenas y de condiciones de sanidad y salubridad ambiental en las comunidades(13).

Con posterioridad, en el informe de 2009 el relator resaltó la preocupación por la situación nutricional de comunidades indígenas colombianas, en especial por la Yukpa (también conocida como Yuko) del resguardo Iroka, en el Departamento del Cesar ${ }^{5}$. Se resalta en el informe que la falta de protección del derecho al territorio y las dificultades en las vías de acceso agravan los problemas de salud de las poblaciones indígenas colombianas(14).

\section{b. Relator especial sobre la situación de los derechos} humanos y las libertades fundamentales de los indigenas

En el informe de 2004 respecto del Estado colombiano, señaló que el conflicto armado que se vive en el país ha generado graves consecuencias sobre

${ }^{5}$ El 91\% de la población padece desnutrición crónica. 
las poblaciones indígenas, afectando en general la realización de sus derechos humanos. Sobre el derecho a la salud en particular, mencionaba que esta situación había cobrado la vida de médicos tradicionales de las comunidades y había reducido la capacidad de estos pueblos para cosechar plantas y productos tradicionales de su entorno natural para el cuidado de la salud. Igualmente, señaló que el avance legal en materia de protección de los pueblos indígenas está desfasado en relación con la precaria situación que viven estos pueblos en el país(15).

En el último informe sobre Colombia, el relator resalta los avances del Estado hacia la ampliación en la cobertura de los servicios de salud, la inclusión de investigación sobre medicina tradicional como línea de política en el Plan de Salud Pública 2007-2010 y la búsqueda de la conformación intercultural de las Entidades Promotoras de Sa$\operatorname{lud}(16)$.

Sin embargo, en el mismo informe enfatiza que en Colombia la población indígena no goza de los mismos estándares de accesibilidad y calidad en salud, si se compara con la mayoría de la población, situación que se ve agravada por las consecuencias del conflicto armado, pues fenómenos como los bloqueos a las vías de transporte y los continuos señalamientos y amenazas a la población civil, impiden el acceso de los PIs a los servicios de salud y también la realización de sus prácticas medicinales, afectando gravemente la realización efectiva de sus derechos económicos, sociales y culturales(16).

\section{Comité de Derechos Económicos, Sociales y Culturales}

En el último informe sobre Colombia presentado al Consejo Económico y Social se resalta que, entre otros grupos vulnerables, los pueblos indígenas tienen grandes dificultades de acceso a los servicios de salud, lo que ha generado mayores tasas de mortalidad infantil en esta población(17).

Los informes de Naciones Unidas, en relación con el derecho a la salud de los pueblos indígenas, aunque reflejan avances por parte de los Estados —entre ellos Colombia—, al proyectar políticas diferenciadas hacia grupos étnicos se indica que aún persisten deficiencias en relación con el acceso a servicios de salud y la promoción de sus prácticas de medicina tradicional.

\section{El derecho a la salud de los PIs desde su propia voz}

Los pueblos indígenas han tenido necesidad de reclamar y pelear por sus derechos, lo que les ha obligado a establecer documentos escritos (leyes, mandatos, cartas, etc.) para este fin. A partir de ellos se puede identificar su cosmovisión respecto de temas como los derechos humanos. Paralelamente se encuentran diversos estudios etnográficos y antropológicos que han aportado importantes elementos en este proceso de construcción de su cosmovisión.

La concepción de derechos humanos de los pueblos indígenas se encuentra en su propia visión del mundo, es decir su cosmovisión, que corresponde a su derecho consuetudinario, llamado también "derecho mayor" o "derecho propio". Cabe resaltar que pueden existir tantos sistemas jurídicos propios como grupos étnicos hayan(18).

De acuerdo con los talleres y grupos focales que se llevaron a cabo con representantes de algunas comunidades indígenas(19), se pudo observar que esta concepción responde a una visión holística e interdependiente de los derechos humanos, de forma que no existe una clasificación generacional de estos derechos, sino que son parte de una noción de armonía y equilibrio del territorio en el que habitan.

En el caso de la salud, los pueblos indígenas la entienden como un estado que va más allá de la ausencia de enfermedad y su relación con la curación, de forma que se percibe como "el producto de la relación armónica entre la naturaleza, los seres humanos y el mundo espiritual. Al romperse alguno de estos tres nexos, el cuerpo se enferma, de modo que se afecta tanto el individuo como la familia entera... Para contrarrestar la enfermedad, es necesario restaurar la armonía perdida buscando el favor de los espíritus de la naturaleza 
y recurriendo a las propiedades medicinales de las plantas"(20).

El Estado colombiano, mediante la Ley 100 de 1993, modificada por la Ley 1.438 de 2011, que regula el sistema de seguridad social en salud, hizo un avance importante al incluir como principio del sistema general el enfoque diferencial, con el fin de reconocer las características particulares de ciertos grupos poblacionales especiales, como los indígenas, y así eliminar situaciones de discriminación y marginación(21).

A pesar de este avance, los pueblos indígenas colombianos, a través de la Organización Nacional Indígena Colombiana (ONIC) y de sus representantes ante el Congreso de la República, han manifestado sus necesidades en materia del reconocimiento y realización del derecho a la salud de sus pueblos. Por ejemplo, en el marco del VII Congreso de los Pueblos Indígenas de la ONIC se aprobó el Mandato General de los pueblos indígenas de Colombia, en el cual manifiestan que el Estado debe generar y consolidar espacios para la promoción y desarrollo de las prácticas de medicina ancestral. En consecuencia, se debe reestructurar la política pública en salud a través de un proceso real de concertación con las autoridades de los PIs, con el fin de promover la medicina tradicional y la aplicación de los Planes de Vida o Planes de Salud Propios(22).

Acorde con la información obtenida a partir de los grupos focales, entre las principales barreras que ven los miembros de los PIs en materia de acceso al sistema de salud colombiano están el idioma y la no disponibilidad de medicamentos generados a partir de los conocimientos tradicionales (19).

Para afrontar esta situación, los representantes de los PIs ante el Congreso de la República, mediante el Proyecto de Ley No 238 de 2004, buscaron una reforma al sistema general de salud. En este proyecto se incluyó la concepción integral de salud de los PIs, al expresar que esta consiste en "un estado de armonía y equilibrio que responde a la comunidad, a la integralidad de la cosmovisión y a la territorialidad, y depende de las relaciones entre las personas, la comunidad y la naturaleza; origen de los componentes esenciales de la medicina tradicional. Comprende el fortalecimiento cultural, la autonomía alimentaria, la educación en salud integral, la promoción de la salud, la prevención y atención de la enfermedad, elementos que garantizan el acceso a los saberes y prácticas culturales basados en criterios de pluralismo médico, complementariedad terapéutica e interculturalidad"(23).

A partir de estos postulados, los PIs consideran que en un Estado pluriétnico, como el colombiano, debe existir un sistema de salud con planes y programas dirigidos a los pueblos indígenas, fundamentados en el respeto por la diversidad étnica y cultural, el reconocimiento de sus instituciones propias y la aplicación efectiva del consentimiento previo, libre e informado, tendiente al fortalecimiento de sus derechos a la medicina indígena, la autonomía alimentaria y la educación en salud indígena.

\section{Discusión}

El sistema de salud colombiano tiene como principio general la solidaridad que, de acuerdo con la interpretación de la Corte Constitucional, en su jurisprudencia, "exige la ayuda mutua entre las personas afiliadas, vinculadas y beneficiarias $(. .).(y)$ se manifiesta (...): en primer lugar, (en) el deber de los sectores con mayores recursos económicos de contribuir al financiamiento de la seguridad social de las personas de escasos ingresos" (24). El anterior postulado es la base de la incorporación de la población vulnerable en los planes de atención en salud.

Si se entiende la solidaridad en un sentido amplio, a la manera de Gunson(5), es decir, como la voluntad de tomar seriamente la perspectiva de otros y actuar para fortalecerla, se puede afirmar que, en el campo del derecho a la salud de los PIs, por medio de la aplicación de este principio no solo se los debe incluir en el sistema occidental de los servicios de salud, sino que también fortalecer su propio sistema tradicional, lo cual redundará en la conservación de su identidad y diversidad cultural. 
Existen factores externos a estas comunidades que afectan no solo la salud física de sus miembros, sino que comprometen otras esferas de la salud, como es su relación con el entorno en el que viven. Muestra de ello es que en la mayoría de los países de América Latina las marcadas desigualdades socioeconómicas afectan particularmente a los grupos poblacionales indígenas, por lo que presentan mayores índices de mortalidad y morbilidad infantil, estando además expuestos a precarias condiciones de salud.

Las extremas condiciones de pobreza y la falta de atención efectiva por parte del Estado han agudizado las condiciones de vulnerabilidad y explotación de las mujeres y niños de estos grupos. En consecuencia, la aparición y diseminación de enfermedades afectan no solo el derecho a la salud integral de las comunidades, sino que a su vez incide en el acceso efectivo a otra serie de derechos y en general a su supervivencia como pueblos.

De la revisión realizada, tanto de los documentos de organismos internacionales y nacionales, como de los resultados de los grupos focales, se encuentra que existe un distanciamiento entre las disposiciones jurídicas estatales y la percepción de los PIs respecto del derecho al acceso a los niveles más altos de salud. Esto significa que el sistema de salud colombiano no acoge una visión integral del derecho a la salud de los pueblos indígenas; por consiguiente, se desconocen las recomendaciones que se incluyen en los informes de los relatores especiales sobre esta materia.

Por esta razón, es factible afirmar que el Estado colombiano desconoce el rol del principio bioético de la solidaridad, que consiste en contribuir en el fortalecimiento de los sistemas de salud en el marco del respeto por la diversidad y el pluralismo. Se requiere un escenario en el que los profesionales de la salud tengan un enfoque cultural para la atención de pacientes pertenecientes a pueblos étnicos, pero que a su vez se busque afianzar el sistema de medicina tradicional, como bien lo recomendó la Organización Mundial de la Salud en la Carta de Ottawa de 1986, en la que reconoció la importancia de la reorientación de los servicios de salud para que tomen en cuenta los factores y necesidades culturales(25).

En esta misma dirección, la Organización Panamericana de la Salud ha incluido asuntos multiculturales en sus áreas de trabajo gracias a la adopción de "La iniciativa de Salud de los Pueblos Indígenas en las Américas", en la cual se hace un reconocimiento de la diversidad étnica y cultural, que debe irradiar en la implementación de los modelos de salud(26).

En este sentido, acorde con la DUBDH y la DUDPI, la promoción y protección del derecho al disfrute del más alto nivel posible de salud para los pueblos indígenas debe fundarse en el respeto por las diferencias culturales, especialmente en los usos y prácticas tradicionales para la prevención y tratamiento de enfermedades(27).

Sumado a lo anterior, se debe generar una interacción entre el sistema educativo y el sistema de salud para construir programas de formación en los que, además de fortalecer las prácticas de la medicina tradicional, se permita, acorde con las necesidades y percepciones de la población, el acceso a otros servicios de calidad. Este diálogo intercultural propicia un mayor grado de aceptación de los programas como propios y aumenta su sostenibilidad(28).

Finalmente, la pregunta por la aplicación y el alcance del principio de solidaridad, en relación con el derecho al disfrute de los más altos niveles de salud de los PIs, tiene dos respuestas: una desde el "ser" y otra desde el "deber ser". En el primer caso se observa que no existe una aplicación efectiva de este principio que permita mejorar notablemente las condiciones de salud de los PIs. En el segundo, esto es, en cuanto al deber ser, las comunidades y los Estados deben replantear las estrategias en materia de prevención y promoción de la salud, con el fin de formular y desarrollar propuestas en políticas públicas en salud a partir del trabajo inclusivo y participativo de los pueblos indígenas colombianos, que den aplicación a los postulados de los instrumentos analizados en este artículo y así se busque la construcción de sistemas de salud de calidad, equitativos, justos e inclusivos. 
Pese a la necesidad de conectar los postulados tanto de la declaración de bioética como de los derechos de los PIs, existe una importante restricción en cuanto al carácter imperativo de estos instrumentos, dada su naturaleza jurídica. De ahí que, para dar efectividad a la interpretación realizada sobre el papel del principio de solidaridad en la promoción del derecho a la salud de los PIs, se requiere, o bien voluntad política para generar políticas públicas que den fuerza a este principio, o bien creación de instrumentos jurídicos imperativos para los Estados. En todo caso, ninguna de estas alternativas depende de los PIs, a quienes les queda la opción y el deber de ejercer presión en instancias internacionales y ante los gobiernos para la aplicación de las instrumentos internacionales revisados en este articulo, con el fin de lograr el efectivo mejoramiento de sus condiciones de acceso al derecho a la salud en los niveles más altos de calidad. 


\section{Referencias}

1. Snead OC. Bioethics and self-governance: the lessons of the Universal Declaration on Bioethics and Human Rights. J Med Philos 2009; 34(3): 204-222.

2. Andorno R. Human dignity and human rights as a common ground for a global bioethics. J Med Philos 2009; 34(3): 223-240.

3. UNESCO. Declaración Universal sobre Bioética y Derechos Humanos. París: Unesco; 2005.

4. Minkler L, Sweeney S. On the indivisibility and interdependence of basic rights in developing countries. Human Rights Quarterly 2011; 33(2): 351-396.

5. Gunson D. Solidarity and the Universal Declaration on Bioethics and Human Rights. J Med Philos 2009; 34(3): 241-260.

6. Colombia. Congreso de la República. Ley 1450 de 2011. Plan Nacional de Desarrollo 2010-2014. Bogotá: Diario Oficial No 48.102.

7. Piovesan F. Derechos sociales, económicos y culturales y derechos civiles y políticos. Sur - Revista Internacional de Direitos Humanos 2004; 1(1): 21-47.

8. Asamblea General de la Organización de las Naciones Unidas. A/RES/61/295. Declaración de las Naciones Unidas sobre los Derechos de los Pueblos Indígenas. París: 2007.

9. Vásquez J. El derecho a la salud. Revista del Instituto Interamericano de Derechos Humanos 2004; 40: 265289.

10. Gevers S. The Right to Health Care. The European Journal of Health Law 2004; 11: 29-34.

11. Organización de las Naciones Unidas. Comité de Derechos Económicos Sociales y Culturales. E/C.12/2000/4. Observación General No. 14. Ginebra: 2000.

12 Grover A. Doc A/64/272. Informe del Relator Especial sobre el derecho de toda persona al disfrute del más alto nivel posible de salud fisica y mental. Nueva York: Asamblea General de la Organización de las Naciones Unidas; 2009.

13. Hunt P. Doc A/59/422. Informe del Relator Especial de la Comisión de Derechos Humanos sobre el derecho de toda persona al disfrute del más alto nivel posible de salud fisica y mental. Asamblea General de la Organización de las Naciones Unidas. Nueva York: 2004.

14. Hunt P. Doc A/HRC/11/12/Add.1. Report of the Special Rapporteur on the right of everyone to the enjoyment of the highest attainable standard of physical and mental health. New York: United Nations General Assembly; 2009.

15. Stavenhagen R. Doc E/CN.4/2005/88/Add.2. Informe del Relator Especial sobre la situación de los derechos humanos y las libertades fundamentales de los indígenas: misión Colombia. Nueva York: 2004.

16. Anaya J. Doc A/HRC/15/34. Informe del Relator Especial de Naciones Unidas sobre la situación de los derechos humanos y las libertades fundamentales de los indígenas: La situación de los pueblos indígenas en Colombia: seguimiento a las recomendaciones hechas por el Relator Especial anterior. Ginebra: Organización de las Naciones 
La solidaridad y las declaraciones internacionales de derechos humanos y bioética - Diana Bernal

Unidas; 2010.

17. Organización de las Naciones Unidas, Consejo Económico y Social. Doc E/C.12/COL/CO/5. Examen de los informes presentados por los Estados Partes de conformidad con los articulos 16 y 17 del Pacto. Ginebra: 2010.

18. Stavenhagen R. Derecho Indígena y Derechos Humanos en América Latina. Costa Rica: Instituto Interamericano de Derechos Humanos; 1988.

19. Bernal D. Grupos Focales Proyecto Perspectiva bioética de la salud y su relación con otros derechos humanos en paises en vía de desarrollo. Silvia, Cauca (Colombia): 2011.

20. Berche AS, García AM, Mantilla A. Los derechos en nuestra propia voz. Pueblos indígenas y DESC: una lectura intercultural. Bogotá: ILSA; 2006.

21. Colombia. Congreso de la República. Ley 1438 de 2011, Reforma al Sistema de Seguridad Social en Salud. Bogotá: Diario Oficial No 47.957; 2011.

22. ONIC. VII Congreso de los Pueblos Indígenas Mandato General. Bogotá: Onic; 2007.

23. Piñacué JE. Proyecto de Ley 238 de 2004. Por el cual se modifica el Sistema General de Seguridad Social y se adicionan normas especiales a favor de los pueblos indigenas. Bogotá: 2004.

24. Escobar R. Sentencia C-111/06. Análisis de constitucionalidad del artículo 47 de la Ley 100 de 1993. Bogotá: Corte Constitucional; 2006.

25. Organización Panamericana de la Salud. Carta de Ottawa para la promoción de la salud. Ottawa: 2010.

26. Organización Panamericana de la Salud. La iniciativa de salud de los pueblos indígenas en las Américas. Winnipeg: 2009.

27. Hunt P. Doc A/HRC/7/11. Informe del Relator Especial sobre el derecho de toda persona al disfrute del más alto nivel posible de salud física y mental. Ginebra: Organización de las Naciones Unidas, Consejo de Derechos Humanos; 2008.

28. United Nations Population Fund. Culture Matters-Working with communities and faith-based organizations: Case studies from Country Programmes. New York: UNFPA; 2004.

Recibido: 7 de mayo de 2012

Aceptado: 18 de agosto de 2012 